\title{
Elevated vitreous Lipocalin-2 levels of patients with proliferative diabetic retinopathy
}

\author{
Hui Wang ${ }^{1,2}$, Hui Lou', Yongrong Li ${ }^{2}$, Fengtao Ji ${ }^{2}$, Wei Chen ${ }^{3}$, Qianyi Lu ${ }^{4}$ and Guoxu Xu ${ }^{1 *}$ (D
}

\begin{abstract}
Background: Lipocalin-2 (LCN2) is a novel adipokine with potential roles in obesity, insulin resistance, and inflammation. This study aims to assess the concentrations of LCN2 and vascular endothelial growth factor (VEGF) expressed in the vitreous humors of patients with proliferative diabetic retinopathy (PDR).

Methods: The concentrations of LCN2 and VEGF were measured from the vitreous of 67 patients undergoing vitrectomy (20 controls and 47 PDR) via enzyme-linked immunosorbent assay (ELISA). Patients with non-ocular pathology that could elevate the LCN2 level in the vitreous were excluded. PDR activity and a history of panretinal photocoagulation were used for further grouping analysis.

Results: The vitreous concentration of LCN2 was statistically significantly higher in the PDR group compared to the control group $(63,522(30,009) \mathrm{pg} / \mathrm{ml}$ versus $1663(1191) \mathrm{pg} / \mathrm{ml}$, respectively; $P<0.001)$. VEGF level was also significantly higher in the PDR group than in the control group (1038 (1326) pg/ml versus $9 \mathrm{pg} / \mathrm{ml}$, respectively; $P<0.001)$. The mean vitreous LCN2 and VEGF levels in active PDR patients were significantly higher than that of the inactive PDR patients. The mean LCN2 concentration in vitreous humor was significantly lower in the 28 PDR patients with a history of complete PRP $(37,304(16,651) \mathrm{pg} / \mathrm{mL})$ in comparison with 19 PDR patients without preperformed panretinal photocoagulation or with preperformed incomplete panretinal photocoagulation (79,796 $(24,391) \mathrm{pg} / \mathrm{mL})$. A significant correlation between the vitreous LCN2 level and VEGF level was found in patients with PDR $(R=0.34 ; P=0.019)$.
\end{abstract}

Conclusions: This report shows a significant increase of LCN2 in the vitreous fluid of patients with PDR and present a significant correlation between LCN2 and VEGF, suggesting LCN2 might be involved in the pathogenesis of PDR.

Keywords: Diabetic retinopathy, Vascular endothelial growth factor, Lipocalin-2

\section{Background}

Diabetic retinopathy (DR) is a serious sightthreatening complication of diabetes. In the USA, studies estimate that $28.5-40.3 \%$ of patients with type 2 diabetes had DR, and $4.4-8.2 \%$ of DR is vision threatening [1]. In a Japanese study, the incidence of

\footnotetext{
* Correspondence: phacoxu@126.com

'Department of Ophthalmology, The Second Affiliated Hospital of Soochow University, 1055 Sanxiang Road, Suzhou 215004, China

Full list of author information is available at the end of the article
}

DR was $26.6 \%$ in patients with type 2 diabetes who did not have DR at baseline over 8 years, and $15.0 \%$ of mild non-proliferative DR showed progression to proliferative DR (PDR) [2].

Chronic low-grade subclinical inflammation in the state of persistent hyperglycemia has an effect on retinal microvascular and eventually leads to DR. [3] Aberrant neovascularization following retinal ischemia is a clinical feature of PDR, which can lead to vitreous hemorrhage and tractional retinal detachment, thus affecting vision

(c) The Author(s). 2020 Open Access This article is licensed under a Creative Commons Attribution 4.0 International License, which permits use, sharing, adaptation, distribution and reproduction in any medium or format, as long as you give appropriate credit to the original author(s) and the source, provide a link to the Creative Commons licence, and indicate if changes were made. The images or other third party material in this article are included in the article's Creative Commons licence, unless indicated otherwise in a credit line to the material. If material is not included in the article's Creative Commons licence and your intended use is not permitted by statutory regulation or exceeds the permitted use, you will need to obtain permission directly from the copyright holder. To view a copy of this licence, visit http://creativecommons.org/licenses/by/4.0/ The Creative Commons Public Domain Dedication waiver (http://creativecommons.org/publicdomain/zero/1.0/) applies to the data made available in this article, unless otherwise stated in a credit line to the data. 
[4]. Several studies have indicated that inflammatory mediators were involved in DR, and inflammatory cells produced angiogenic cytokines and growth factors, which were implicated in the progression of DR [5-7].

Lipocalin-2 (LCN2), also known as neutrophil gelatinase-associated lipocalin (NGAL), is released by various cell types that has been found to be involved in multiple processes such as metabolic homeostasis, apoptosis, infection, immune response, or inflammation [8]. In the existing literature, elevated LCN2 was also detected in the aqueous humor of patients with idiopathic acute anterior uveitis [9] and central retinal vein occlusion [10] and vitreous fluid from patients with rhegmatogenous retinal detachment [11]. A large number of studies indicate that the level of LCN2 is closely related to the degree of inflammation in various inflammatory diseases [12-17]. However, LCN2 level in vitreous fluid and the relation with other inflammatory cytokines such as vascular endothelial growth factor (VEGF) in diabetic patients with DR or PDR remain unclear. Therefore, the present study was aimed to investigate a possible correlation between LCN2 and VEGF levels in the vitreous of eyes with PDR to provide supporting evidence of LCN2's potential involvement in the progress of PDR and investigate the potential ability of LCN2 as biomarker of PDR.

\section{Methods}

\section{Study population}

This study enrolled 67 patients: 47 diabetic patients with PDR had vitrectomies for vitreous hemorrhage or tractional retinal detachment and a control group of 20 non-diabetic patients had vitrectomies for idiopathic macular holes, only one eye from each patient being included. Patients with non-ocular pathology that could elevate the LCN2 level in the vitreous such as cardiovascular diseases, intestinal inflammation, breast cancer, pancreatic diseases, etc. were excluded. Samples from eyes obtained during a repeat vitrectomy were excluded. Table 1 presents the backgrounds and demographics of the 67 patients. This study was conducted in accordance with the Declaration of Helsinki and written informed consent was obtained from all patients. The study was approved by the ethical committee of The Second Affiliated Hospital of Soochow University.

PDR was classified as active and inactive. Inactive PDR was defined as the presence of nonvascularized fibrotic epiretinal membranes, and was present in 15 patients. Active PDR was defined as visible large new vessels within the proliferative tissue, and was present in $32 \mathrm{pa}-$ tients. The clinical information of patients' eyes was recorded during the operation, and the operative videotapes were kept for subsequent analysis. These data were reviewed by three ophthalmologists independently
Table 1 Intravitreous Concentrations of LCN2 and VEGF in the PDR and Control Groups

\begin{tabular}{lllc}
\hline & PDR Group & Control Group & $P$ \\
\hline No. of cases & 47 & 20 & \\
Gender (male: female) & $16: 31$ & $7: 13$ & 0.82 \\
Age (years) & $61.32(7.22)$ & $59.85(6.90)$ & 0.38 \\
HbAlc (\%) & $8.16(2.44)$ & $5.03(0.51)$ & $<0.001$ \\
VH & 32 & - & \\
TRD & 6 & - & \\
VH + TRD & 9 & - & \\
Idiopathic macular hole & - & 20 & $<0.001$ \\
LCN2 (pg/ml) & $63,522(30,009)$ & $1663(1191)$ & $<0.001$ \\
VEGF (pg/ml) & $1038(1326)$ & $<9^{\mathrm{a}}$ & \\
\hline
\end{tabular}

LCN2 Lipocalin-2, VEGF vascular endothelial growth factor; $P D R$ proliferative diabetic retinopathy, HbAlc glycosylated hemoglobin, $V H$ vitreous hemorrhage, $T R D$ tractional retinal detachment

${ }^{a}$ Vitreous level of VEGF regarded as $9 \mathrm{pg} / \mathrm{mL}$ in control participants

to avoid a bias before further testing of LCN2 and VEGF concentrations in vitreous fluid.

\section{Sample collection}

Vitrectomy was performed using a standard three-port 25-gauge PPV technique. Undiluted vitreous samples $(0.6$ to $1.0 \mathrm{ml})$ were aspirated from the mid vitreous at the initial stage of vitrectomy, and were placed on ice in sterile tubes (Eppendorf) immediately. The tubes were then centrifuged at $13,000 \mathrm{rpm}$ for $5 \mathrm{~min}$ at $4{ }^{\circ} \mathrm{C}$ within $30 \mathrm{~min}$. The supernatant was dispensed as $200 \mu \mathrm{L}$ aliquots and stored at $-80^{\circ} \mathrm{C}$ until analysis was performed. Enzyme-linked immunosorbent assay (ELISA) was used to determine the concentration of LCN2 or VEGF in the samples.

\section{Measurement of LCN2 and VEGF}

LCN2 levels were analyzed using a commercially available ELISA kit (BioVendor, Brno, Czech Republic) according to the manufacturer's instructions. Recombinant LCN2 was serially diluted to obtain a standard graph by a standard method. The analyses were made in duplicate for each sample. The same method was used to detect VEGF in vitreous fluid with ELISA kit of human VEGF (R\&D Systems, Minneapolis, Minnesota, USA).

\section{Statistical analysis}

SPSS 17.0 software (SPSS Inc., Chicago, IL, USA) was used to perform the statistical analyses. Data are expressed as means (standard deviation). For quantitative variables, differences between two groups were determined by Student's $\mathrm{t}$ test (2-tailed). The primary outcome of this study was to compare the vitreous concentrations of LCN2 and VEGF of PDR patients with non-diabetic patients. The secondary outcomes were the 
comparison of LCN2 and VEGF concentrations between inactive PDR and active PDR, and the comparison between eyes with and without complete panretinal photocoagulation (PRP). Correlation between the concentration of vitreous LCN2 and VEGF was quantified using the Pearson correlation coefficient. The differences were considered significant at $P<0.05$.

\section{Results}

Table 1 summarizes the surgical indications of PDR patients, including 32 eyes of vitreous hemorrhage, 6 eyes of tractional retinal detachment, and 9 eyes of vitreous hemorrhage with tractional retinal detachment. Hemoglobin in vitreous had a significant effect on the results. To prevent this, patients with recent vitreous hemorrhage ( $<1$ month) were not enrolled in this study.

The vitreous levels of LCN2 were significantly higher in the PDR group than in the control group $(63,522$ $(30,009) \mathrm{pg} / \mathrm{ml}$ versus 1663 (1191) $\mathrm{pg} / \mathrm{ml}$, respectively; $P<0.001)$ (Fig. 1a). VEGF levels were also significantly higher in the PDR group than in the control group (1038 (1326) $\mathrm{pg} / \mathrm{ml}$ versus $9 \mathrm{pg} / \mathrm{ml}$, respectively; $P<0.001$ ) (Fig. 1b). In fact, VEGF was not detected in patients with idiopathic macular hole, and $9 \mathrm{pg} / \mathrm{ml}$ represents the sensitivity limit of the detection kit.

We further divided PDR patients (47 eyes) into active PDR (32 eyes) and inactive PDR (15 eyes). The mean vitreous level of LCN2 was $78,465(23,637) \mathrm{pg} / \mathrm{ml}$ in eyes of patients with active PDR and $31,645(11,122) \mathrm{pg} / \mathrm{ml}$ in eyes of patients with inactive PDR. The mean vitreous LCN2 level in active PDR patients was significantly higher than that of the inactive PDR patients $(P<0.001$; Fig. 2a). The mean vitreous level of VEGF was 1419.2 (1461) $\mathrm{pg} / \mathrm{ml}$ in the eyes with active PDR and 224 (69) $\mathrm{pg} / \mathrm{ml}$ in the eyes with inactive PDR. This difference was statistically significant $(P<0.001$, Fig. $2 b)$. Our findings also demonstrated a significant correlation between the vitreous LCN2 level and VEGF level in patients with PDR ( $\mathrm{R}=0.34 ; P=0.019$; Fig. 3 ).

The effect of PRP on the vitreous levels of LCN2 and VEGF was also evaluated in our study. Of 47 patients with PDR, 28 (57.4\%) were performed with complete PRP (1500 to 3000 spots of burns) 17.2 (20.1) months (2-46) before vitrectomy. The mean LCN2 concentration in vitreous humor was lower in the 28 PDR patients with a history of complete PRP $(37,304(16,651) \mathrm{pg} / \mathrm{mL})$ in comparison with 19 PDR patients without preperformed PRP or with preperformed incomplete PRP (79, $796(24,391) \mathrm{pg} / \mathrm{mL})$ (Fig. 4a). This difference was considered statistically significant $(P<0.001)$. We also analyzed the relationship between the duration from PRP to vitrectomy and the concentration of LCN2 and VEGF in the vitreous of all 28 PDR patients preperformed with complete PRP. Pearson's correlation analysis indicated that there was no significant correlation between the duration from PRP to vitrectomy and the vitreous level of LCN2 or VEGF $(P=0.139$ and $P=0.205$, respectively).

\section{Discussion}

The results indicated that vitreous LCN2 and VEGF levels were significantly higher in the PDR group compared to the nondiabetic control group. To our knowledge, this is the first study in the literature evaluating vitreous LCN2 in patients with PDR. Although whether PDR in this study was in active phase depended on our clinical experience, there might be some mistakes, but it does not affect our conclusion that vitreous LCN2 in active PDR was significantly higher than that in inactive

\section{A) Lipocalin-2}

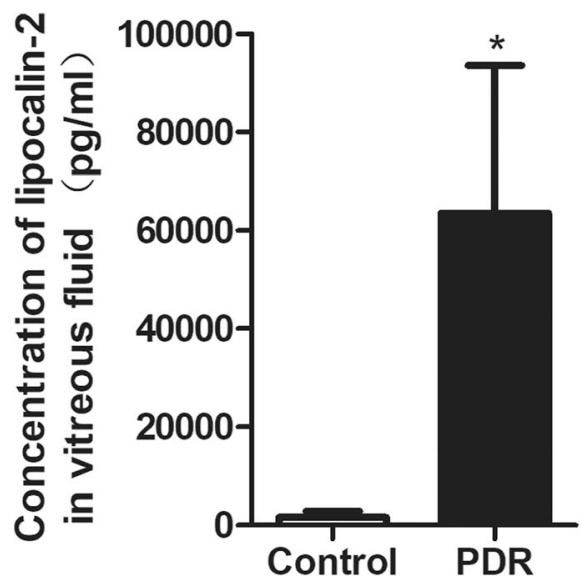

B) VEGF

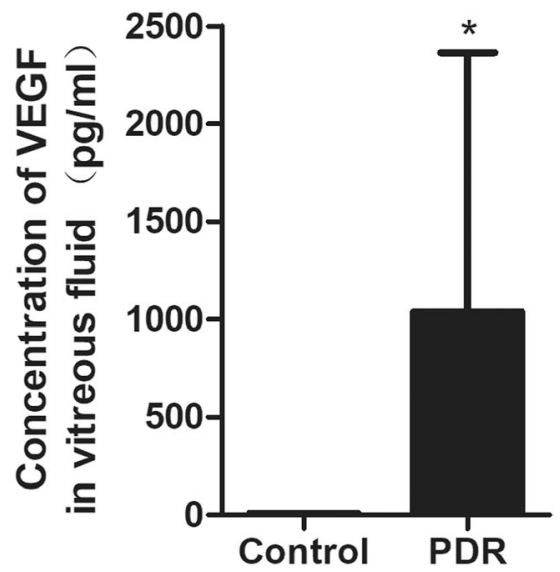

Fig. 1 a Vitreous LCN2 levels in eyes with nondiabetic control group and eyes with PDR. b Vitreous vascular endothelial growth factor (VEGF) level in eyes nondiabetic control group and eyes with PDR. PDR = proliferative diabetic retinopathy. ${ }^{*} P<.0001$ 

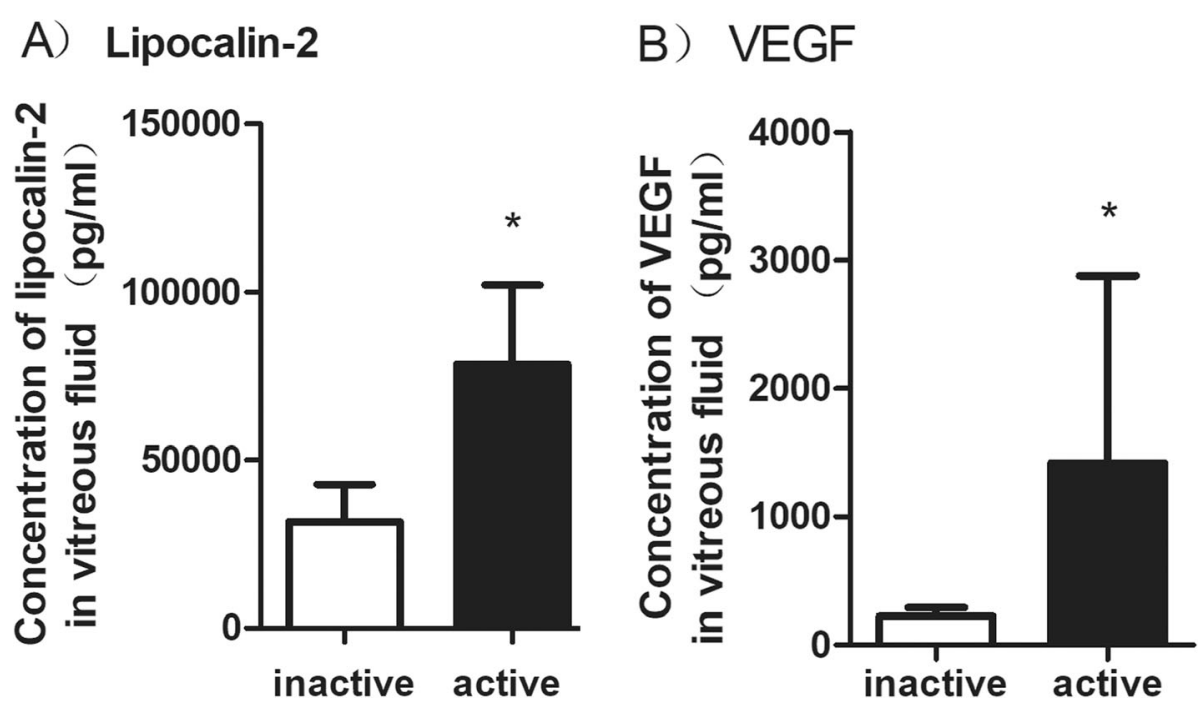

Fig. 2 a Lipocalin-2 levels in vitreous samples of active and inactive PDR. b Vascular endothelial growth factor (VEGF) levels in vitreous samples active and inactive PDR. PDR $=$ proliferative diabetic retinopathy. ${ }^{*} P<.0001$

PDR. These findings suggest that LCN2 may be involved in the vascularization of PDR.

LCN2 has been identified as a potential biomarker for several common inflammatory diseases including acute kidney injury, lupus nephritis, cardiovascular diseases, or intestinal inflammation [18]. Although LCN2 is highly upregulated under a large number of inflammatory conditions, both pro- and anti-inflammatory properties of this adipokine have been reported. Hu et al. described that LCN2 upregulation protects hepatocytes from interleukin-1beta-induced inflammation [19]. Vichaya found that LCN2 is dispensable for sterile inflammationinduced sickness and depression-like behavior [16].
Recently, LCN2 was also found to be involved in the pathogenesis of Alzheimer's disease [20]. In terms of ophthalmic diseases, the work of Parmar et al. have revealed that $\mathrm{LCN} 2$ plays a regulatory role in retinal inflammation during retinal degeneration such as Stargardt, retinitis pigmentosa and age-related macular degeneration. They found that LCN2 induced expression of antioxidant enzymes heme oxygenase 1 and superoxide dismutase 2 in RPE cells and could inhibit the cytotoxic effects of $\mathrm{H}_{2} \mathrm{O}_{2}$ and LPS, and exacerbated inflammation following light exposure was observed in LCN2 knockout mice model of Stargardt disease and age-related macular degeneration [21]. In the study of

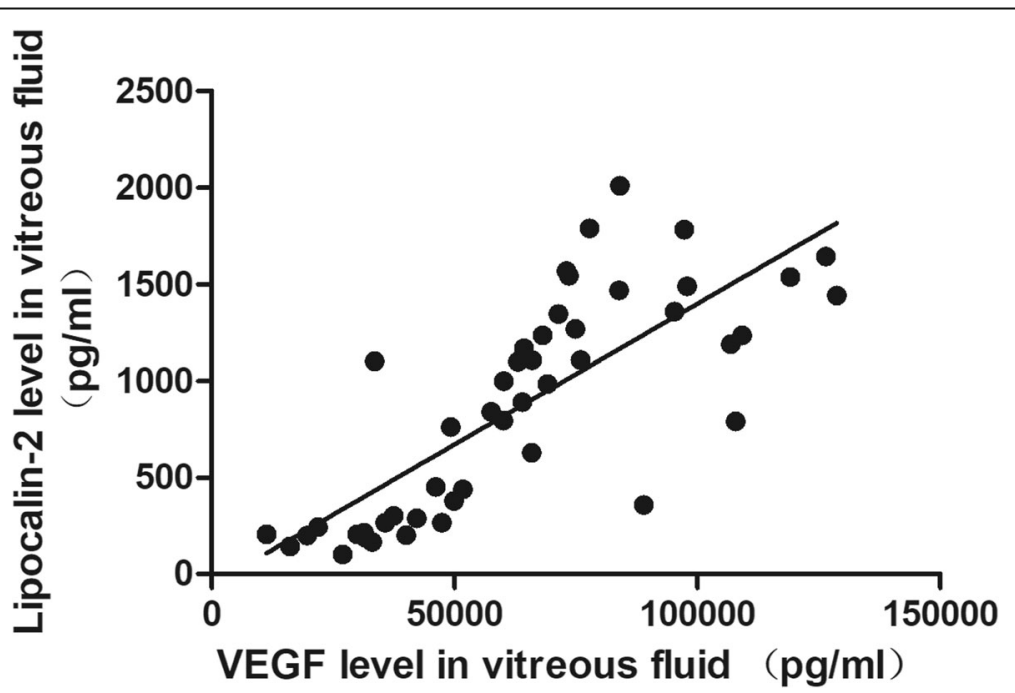

Fig. 3 A linear relationship between Lipocalin-2 and vascular endothelial growth factor (VEGF) in vitreous fluid with proliferative diabetic retinopathy. [Pearson correlation coefficient] $\mathrm{R}=0.34 ; P=0.019$ 


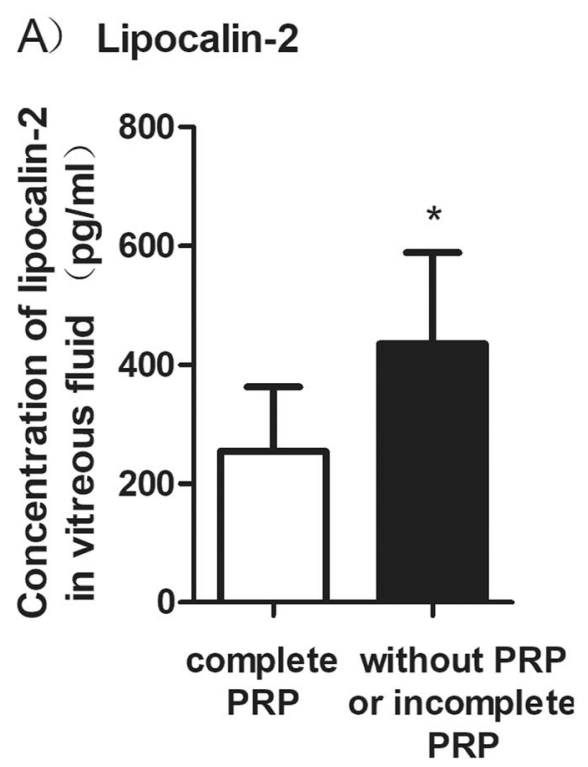

\section{B) VEGF}

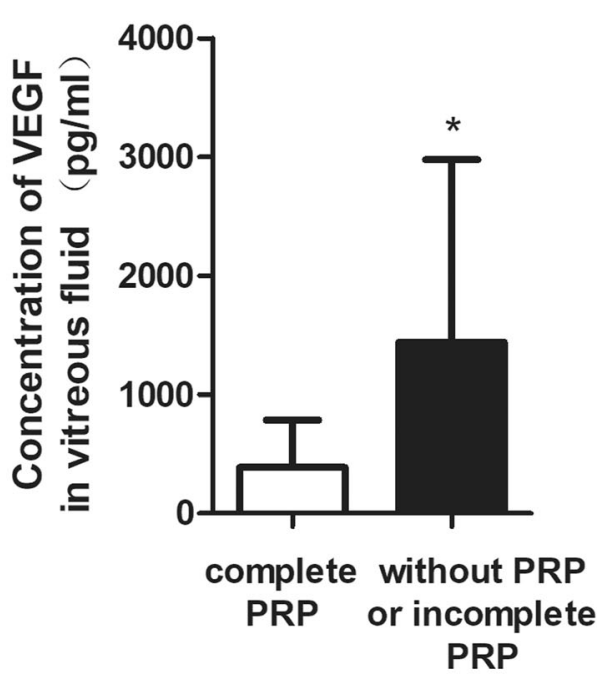

Fig. 4 a Vitreous LCN2 levels in 28 PDR eyes with preperformed complete PRP and 19 PDR eyes without preperformed PRP or with preperformed incomplete PRP. $\mathbf{b}$ Vascular endothelial growth factor (VEGF) levels in 28 PDR eyes with preperformed complete PRP and 19 PDR eyes without preperformed PRP or with preperformed incomplete PRP. PDR = proliferative diabetic retinopathy; PRP = panretinal photocoagulation. ${ }^{*} P<.0001$

the Yaran Koba group, they demonstrated that increased LCN2 levels in the eyes of patients with central retinal vein occlusion begin an intense neuroinflammatory process and cause iron accumulation in retinal cells which may lead to the complications such as macular edema, macular ischemia, and neovascularization [10]. In a recent study, it has been demonstrated that a positive correlation between vitreous levels of LCN2 and proliferative vitreoretinopathy grading in patients with rhegmatogenous retinal detachment, revealing a potential role in the pathogenesis and progression of proliferative vitreoretinopathy. They thought LCN2 seems to play a role in reactive gliosis and neuroinflammation, which are involved in PVR pathophysiology.

At present, VEGF is recognized as an important protein in the pathogenesis of PDR, and anti-VEGF therapy has achieved good results in clinical use. Not surprisingly, we also found a significant increase in intravitreal VEGF concentration in PDR patients. The mean concentration of vitreous VEGF in eyes with PDR in our study was similar with that in other studies [22, 23]. Previous studies have found that successful PRP can effectively reduce the concentration of VEGF in the vitreous. Our study found that the concentrations of LCN2 and VEGF in vitreous fluid of PDR patients who had completed PRP were significantly lower than those of PDR patients who had not completed PRP. This indicates that PRP might affects the production of LCN2 and VEGF simultaneously. Similar results were obtained when comparing the concentrations of LCN2 and VEGF in vitreous fluid of inactive PDR with active PDR. This suggests that both LCN2 and VEGF may be related to the activity of neovascularization. The exact mechanisms of PRP are unclear, but it is possible that the decreased area of retinal tissue leads to improved oxygenation and a reduction in the levels of VEGF. A reduction in levels of VEGF may be important in reducing the risk of harmful new vessels forming. In our study, PRP reduced LCN2 concentration in vitreous cavity, the reason may be that PRP destroyed retinal tissues and reduced LCN2 secretion.

The significant correlation between the vitreous LCN2 and VEGF levels in eyes with PDR suggests that there may be upstream-downstream relationship between the two proteins. Yang et al. previously reported that VEGF was significantly increased with LCN2 expression in MCF-7 human breast cancer cells [24]. They found LCN2-induced VEGF was mediated through hypoxiainducible factor 1 alpha $(\mathrm{HIF}-1 \alpha)$. Intravitreous VEGF and HIF- $1 \alpha$ in diabetic patients with PDR were found increased and related mutually previously [25]. Moreover, the production of VEGF was diminished in a diabetic mice model lacking HIF-1 $\alpha$ expression [26]. These findings clearly support the hypothesis that LCN2 upregulates VEGF expression through HIF-1 $\alpha$ and promotes neovascularization in PDR.

The limitation of this study is that the number of eyes in the study group and the control group was unbalanced at baseline, but the statistical value $P$ in our primary outcome was less than 0.001 , which does not seem to affect our conclusion that the concentration of LCN2 
and VEGF in the vitreous cavity of PDR patients is higher than that of the control group.

\section{Conclusions}

In conclusion, this study provides evidence that the levels of both LCN2 and VEGF are elevated in the vitreous fluid from patients with PDR compared to non diabetic patients. Although our study demonstrates a significant correlation between LCN2 and VEGF levels, it does not establish a definitive cause and effect relationship between LCN2 and VEGF, and further mechanism research will be needed. Our findings indicate the potential role of LCN2 in the development and progression of PDR. Further studies will be required to confirm the possible molecular mechanisms of LCN2 that contribute to inflammatory response and retinal angiogenesis in animal models of DR, which will help to determine the precise role of LCN2 as a potential PDR biomarker or therapeutic target.

\section{Abbreviations \\ LCN2: lipocalin-2; VEGF: vascular endothelial growth factor; PDR: proliferative diabetic retinopathy; ELISA: enzyme-linked immunosorbent assay; HbAlc: glycosylated hemoglobin; VH: vitreous hemorrhage; TRD: tractional retinal detachment; PRP: panretinal photocoagulation}

\section{Acknowledgements}

Not applicable.

\section{Authors' contributions}

$\mathrm{WH}$ has collected data and has been involved in drafting the manuscript. $\mathrm{LH}$ have collected data and helped revising the draft critically for important intellectual content. LYR, JFT, CW, and LQY have made substantial contributions in analysis and interpretation of data and revising the draft critically for important intellectual content. XGX contributed to data analysis and interpretation and helped revising the draft critically for important intellectual content. All the authors read and approved the final manuscript and agreed to be accountable for all aspects of the work in ensuring that questions related to accuracy or integrity of any part of the work are appropriately investigated and resolved.

\section{Funding}

This work was supported by National Natural Science Foundation of China (No:81700855); the National Natural Science Foundation of China (No. 81700847) and the Natural Science Foundation of Jiangsu Province (No BK20170365). However, the funders had no role in the design of the study, data collection, and analysis, interpretation of data, preparation of the manuscript or decision to publish.

\section{Availability of data and materials}

The datasets used and analysed in the current study are available from the corresponding author on reasonable request.

\section{Ethics approval and consent to participate}

The study was conducted in accordance with the Declaration of Helsinki and written informed consent was obtained from all patients. The study was approved by the ethical committee of The Second Affiliated Hospital of Soochow University.

\section{Consent for publication}

Not applicable.

\section{Competing interests}

The authors declare that they have no competing interests.

\section{Author details}

'Department of Ophthalmology, The Second Affiliated Hospital of Soochow University, 1055 Sanxiang Road, Suzhou 215004, China. ${ }^{2}$ Department of Ophthalmology, The Second People's Hospital of Hefei, Hefei 230011, China. ${ }^{3}$ Department of Ophthalmology, Affiliated Hospital of Nantong University, Nantong 226001, Jiangsu, China. ${ }^{4}$ Department of Ophthalmology, The First Affiliated Hospital of Soochow University, Suzhou 215006, China.

Received: 20 February 2020 Accepted: 1 May 2020

Published online: 30 June 2020

\section{References}

1. Zhang $X$, Saaddine JB, Chou CF, et al. Prevalence of diabetic retinopathy in the United States, 2005-2008[J]. JAMA. 2010;304(6):649-56.

2. Kawasaki R, Tanaka S, Tanaka S, et al. Incidence and progression of diabetic retinopathy in Japanese adults with type 2 diabetes: 8 year follow-up study of the Japan diabetes complications study (JDCS). Diabetologia. 2011;54(9): 2288-94.

3. Gouliopoulos NS, Kalogeropoulos C, Lavaris A, et al. Association of serum inflammatory markers and diabetic retinopathy: a review of literature. Eur Rev Med Pharmacol Sci. 2018;22(21):7113-28.

4. Gologorsky D, Thanos A, Vavv as D. Therapeutic interventions against inflammatory and angiogenic mediators in proliferative diabetic retinopathy. Mediat Inflamm. 2012;2012:629452

5. Spencer BG, Estevez JJ, Liu E, et al. Pericytes, inflammation, and diabetic retinopathy. Inflammopharmacol. 2019.

6. Youngblood $H$, Robinson $\mathrm{R}$, Sharma A et al. Proteomic Biomarkers of Retinal Inflammation in Diabetic Retinopathy. Int J Mol Sci 2019; 20(19):4755 e1-19.

7. Biswas S, Sarabusky M, Chakrabarti S. Diabetic Retinopathy, IncRNAs, and Inflammation: A Dynamic, Interconnected Network. J Clin Med 2019; 8(7): 1033e1-26.

8. Li D, Yan Sun W, Fu B, et al. Lipocalin-2-the myth of its expression and function. Basic Clin Pharmacol Toxicol. 2019

9. Tang W, Ma J, Gu R, et al. Lipocalin 2 suppresses ocular inflammation by inhibiting the activation of NF-kappabeta pathway in endotoxin-induced uveitis. Cell Physiol Biochem. 2018;46(1):375-88.

10. Koban Y, Sahin S, Boy F, et al. Elevated lipocalin-2 level in aqueous humor of patients with central ret inal vein occlusion. Int Ophthalmol. 2019;39(5): 981-6.

11. Batsos G, Christodoulou E, Vartholomatos G, et al. Vitreous levels of Lipocalin-2 on patients with primary rhegmatogenous ret inal detachment. PLoS One. 2019;14(12):e0227266.

12. Shibata K, Sato K, Shirai $R$, et al. Lipocalin-2 exerts pro-atherosclerotic effects as evidenced by in vitro and in vivo experiments. Heart Vessel. 2020.

13. Gumpper K, Dangel AW, Pita-Grisanti V, et al. Lipocalin-2 expression and function in pancreatic diseases. Pancreatology. 2020;20(3):419-24.14.

14. Ghosh S, Stepicheva N, Yazdankhah M, et al. The role of lipocalin-2 in agerelated macular degeneration (AMD). Cell Mol Life Sci. 2020;77(2):1-17.

15. Yu L, Zhou L, Li Q, et al. Elevated urinary lipocalin-2, interleukin-6 and monocyte chemoattractant protein-1 levels in children with congenital ureteropelvic junction obstruction. J Pediatr Urol. 2019;15(1):44 e41-7.

16. Vichaya EG, Gross PS, Estrada DJ, et al. Lipocalin-2 is dispensable in inflammation-induced sickness and depression-like behavior. Psychopharmacology. 2019;236(10):2975-82

17. Zhang Y, Liu J, Yang B, et al. Ginkgo biloba extract inhibits Astrocytic Lipocalin-2 expression and alleviates Neuroinflammatory injury via the JAK2/ STAT3 pathway after ischemic brain stroke. Front Pharmacol. 2018;9:518.

18. Abella V, Scotece M, Conde J, et al. The potential of lipocalin-2/NGAL as biomarker for inflammatory and metabolic diseases. Biomarkers. 2015;20(8): 565-71.

19. Hu Y, Xue J, Yang Y, et al. Lipocalin 2 Upregulation protects hepatocytes from IL1-beta-induced stress. Cell Physiol Biochem. 2015;36(2):753-62.

20. Eruysal E, Ravdin L, Kamel H, et al. Plasma lipocalin-2 levels in the preclinical stage of Alzheimer's disease. Alzheimers Dement (Amst). 2019;11:646-53.

21. Parmar T, Parmar VM, Perusek $L$, et al. Lipocalin 2 plays an important role in regulating inflammation in retinal degeneration. J Immunol. 2018;200(9): 3128-41.

22. Noma H, Funatsu H, Yamashita $H$, et al. Regulation of angiogenesis in diabetic retinopathy: possible balance between vascular endothelial growth factor and endostatin. Arch Ophthalmol. 2002;120(8):1075-80. 
23. Simo R, Lecube A, Segura RM, et al. Free insulin growth factor-I and vascular endothelial growth factor in the vitreous fluid of patients with proliferative diabetic retinopathy. Am J Ophthalmol. 2002;134(3):376-82.

24. Yang J, McNeish B, Butterfield C, et al. Lipocalin 2 is a novel regulator of angiogenesis in human breast cancer. FASEB J. 2013;27(1):45-50.

25. Wang $X$, Wang $G$, Wang $Y$. Intravitreous vascular endothelial growth factor and hypoxia-inducible factor 1a in patients with proliferative diabetic retinopathy. Am J Ophthalmol. 2009;148(6):883-9.

26. Vadlapatla RK, Vadlapudi AD, Mitra AK. Hypoxia-inducible factor-1 (HIF-1): a potential target for intervention in ocular neovascular diseases. Curr Drug Targets. 2013;14(8):919-35.

\section{Publisher's Note}

Springer Nature remains neutral with regard to jurisdictional claims in published maps and institutional affiliations.

Ready to submit your research? Choose BMC and benefit from:

- fast, convenient online submission

- thorough peer review by experienced researchers in your field

- rapid publication on acceptance

- support for research data, including large and complex data types

- gold Open Access which fosters wider collaboration and increased citations

- maximum visibility for your research: over $100 \mathrm{M}$ website views per year

At BMC, research is always in progress.

Learn more biomedcentral.com/submissions 